\title{
Regulations for Confidentiality of Health Records
}

\author{
Kamyar Ghabili ${ }^{1}$, Samad EJ Golzari ${ }^{2 *}$
}

1. Medical Philosophy and History Research Center, Tabriz University of Medical sciences, Tabriz, Iran

2. Liver and Gastrointestinal Disease Research Center, Tabriz University of Medical Sciences, Tabriz, Iran

The emergence of novel health record devices has opened a new avenue for medical sciences. Portable electronic health record devices are of recently-introduced inventions that would definitely contribute to the facilitation of providing health care for the trauma patients in low-resource settings (1). In addition to the benefits these new tools would provide for the patients, hospital workflow will not be hindered by the burden of providing primary physiological data required for the medical management.

However, recently it has been argued that health record data should be considered confidential and rules or guidelines implemented in order to mitigate ethical concerns (2). Undoubtedly, implementation of effective regulations in this regard stands quite necessary. However, these regulations should exempt some fields from obtaining consent from the individuals, especially in the field of critical care medicine. For instance in the intrahospital, perioperative and emergency settings, the physiological data are used quite often to save lives of the patients; yet, no consents are obtained from the patients in this regard. Implementation of such guidelines, though seemingly necessary, should not impede the utilization of physiological or medical health data, especially in the emergency conditions where any delay in the process of data utilization could risk the lives of the individuals.

\section{References}

1. Zargaran E, Schuurman N, Nicol AJ, Matzopoulos R, Cinnamon J, Taulu T, et al. The electronic Trauma Health Record: design and usability of a novel tablet-based tool for trauma care and injury surveillance in low resource settings. J Am Coll Surg. 2014;218(1):41-50. doi: 10.1016/j.jamcollsurg.2013.10.001.

2. Stephen Fairclough. Physiological data must remain confidential. Nature 505, 263. doi:10.1038/505263a

\section{Corresponding author:}

Samad EJ Golzari, MD

Liver and Gastrointestinal Disease Research Center, Tabriz University of Medical Sciences, Golgasht St. 5166614756

Tabriz, Iran

Phone: +989141151894 E-mail: dr.golzari@hotmail.com

Receive date: 2014-04-01| Accept date: 2014-04-29 | Publish date: 2014-05-08

DOI: 10.7575/aiac.abcmed.15.03.01.01 